\title{
THE LEECH EXOPLANET IMAGING SURVEY: ORBIT AND COMPONENT MASSES OF THE INTERMEDIATE-AGE, LATE-TYPE BINARY NO UMa* $†$
}

\author{
Joshua E. Schlieder ${ }^{1,2,14}$, Andrew J. Skemer ${ }^{3}$, Anne-Lise Maire ${ }^{4}$, Silvano Desidera $^{4}$, Philip Hinz ${ }^{3}$, \\ Michael F. Skrutskie ${ }^{5}$, Jarron Leisenring ${ }^{3}$, Vanessa Bailey ${ }^{6}$, Denis Defrère ${ }^{3}$, Simone Esposito ${ }^{7}$, Klaus G. Strassmeier $^{8}$, \\ Michael Weber ${ }^{8}$, Beth A. Biller ${ }^{2,9}$, Mickaël Bonnefoy ${ }^{2,10}$, Esther Buenzlí ${ }^{2}$, Laird M. Close ${ }^{3}$, Justin R. Crepp ${ }^{11}$, \\ Josh A. Eisner ${ }^{3}$, Karl-Heinz Hofmann ${ }^{12}$, Thomas Henning ${ }^{2}$, Katie M. Morzinski ${ }^{3}$, Dieter Scherti ${ }^{12}$, \\ Gerd Weigelt $^{12}$, AND Charles E. WoOdWARD ${ }^{13}$ \\ ${ }^{1}$ NASA Ames Research Center, Space Science and Astrobiology Division, MS 245-6, Moffett Field, CA 94035, USA; joshua.e.schlieder@nasa.gov \\ Max-Planck-Institut für Astronomie, Königstuhl 17, D-69117, Heidelberg, Germany
${ }^{3}$ Steward Observatory, Department of Astronomy, University of Arizona, 933 N. Cherry Avenue, Tucson, AZ 85721, USA \\ ${ }^{4}$ INAF-Osservatorio Astronomico di Padova, Vicolo dell'Osservatorio 5, I-35122, Padova, Italy \\ ${ }^{5}$ Department of Astronomy, University of Virginia, Charlottesville, VA, 22904, USA \\ ${ }^{6}$ Kavli Institute for Particle Astrophysics and Cosmology, Stanford University, Stanford, CA 94305, USA \\ ${ }^{7}$ INAF-Osservatorio Astrofisico di Arcetri, Largo E. Fermi 5, I-50125, Firenze, Italy \\ ${ }^{8}$ Leibniz-Institut für Astrophysik Potsdam (AIP), An der Sternwarte 16, D-14482, Potsdam, Germany \\ ${ }^{9}$ Institute for Astronomy, University of Edinburgh, Blackford Hill, Edinburgh EH9 3HJ, UK \\ ${ }^{10}$ Université Grenoble Alpes, IPAG, 38000, Grenoble, 38000, Grenoble; CNRS, IPAG, F-38000 Grenoble, France \\ ${ }^{11}$ Department of Physics, University of Notre Dame, 225 Nieuwland Science Hall, Notre Dame, IN, 46556, USA \\ ${ }^{12}$ Max-Planck-Institut für Radioastronomie, Auf dem Hügel 69, D-53121, Bonn, Germany \\ ${ }^{13}$ Minnesota Institute for Astrophysics, University of Minnesota, 116 Church Street, SE, Minneapolis, MN, 55455, USA \\ Received 2015 June 29; accepted 2015 October 8; published 2016 February 2
}

\section{ABSTRACT}

\begin{abstract}
We present high-resolution Large Binocular Telescope LBTI/LMIRcam images of the spectroscopic and astrometric binary NO UMa obtained as part of the LBT Interferometer Exozodi Exoplanet Common Hunt exoplanet imaging survey. Our $H_{-}^{-}, K_{s^{-}}$, and $L^{\prime}$-band observations resolve the system at angular separations $<0$ ! 09 . The components exhibit significant orbital motion over a span of $\sim 7$ months. We combine our imaging data with archival images, published speckle interferometry measurements, and existing spectroscopic velocity data to solve the full orbital solution and estimate component masses. The masses of the $\mathrm{K} 2.0 \pm 0.5$ primary and $\mathrm{K} 6.5 \pm 0.5$ secondary are $0.83 \pm 0.02 M_{\odot}$ and $0.64 \pm 0.02 M_{\odot}$, respectively. We also derive a system distance of $d=25.87 \pm 0.02 \mathrm{pc}$ and revise the Galactic kinematics of NO UMa. Our revised Galactic kinematics confirm NO UMa as a nuclear member of the $\sim 500$ Myr old Ursa Major moving group, and it is thus a mass and age benchmark. We compare the masses of the NO UMa binary components to those predicted by five sets of stellar evolution models at the age of the Ursa Major group. We find excellent agreement between our measured masses and model predictions with little systematic scatter between the models. NO UMa joins the short list of nearby, bright, late-type binaries having known ages and fully characterized orbits.
\end{abstract}

Key words: binaries: general - instrumentation: adaptive optics - stars: fundamental parameters - stars: individual (NO UMa) - stars: late-type - techniques: high angular resolution

\section{INTRODUCTION}

Multiple star systems are a natural outcome of the star formation process. Thus, stars in binaries and higher-order systems are prevalent in the solar neighborhood. Raghavan et al. (2010) surveyed more than 450 solar-type stars $\left(M_{*} \approx 0.7-1.1 M_{\odot}\right.$, mid-K to late-F spectral types) in a $25 \mathrm{pc}$ volume around the Sun and found a multiplicity fraction of $41 \pm 3 \%$. The majority of these multiples are binaries (Duchêne \& Kraus 2013). Much less frequent, however, are

\footnotetext{
* The LBT is an international collaboration among institutions in the United States, Italy and Germany. LBT Corporation partners are: The University of Arizona on behalf of the Arizona university system; Istituto Nazionale di Astrofisica, Italy; LBT Beteiligungsgesellschaft, Germany, representing the Max-Planck Society, the Astrophysical Institute Potsdam, and Heidelberg University; The Ohio State University, and The Research Corporation, on behalf of The University of Notre Dame, University of Minnesota and University of Virginia.

$\dagger$ Based on data obtained with the STELLA robotic telescope in Tenerife, an AIP facility jointly operated by AIP and IAC.

${ }^{14}$ NASA Postdoctoral Program Fellow.
}

multiple systems amenable to detailed orbit characterization. Distance, separation, and mass must all be favorable to make the measurement of orbital parameters feasible on reasonable timescales using both spectroscopic and astrometric monitoring. Analyses of such systems provide precise estimates of parameters such as period, eccentricity, inclination, and, most critically, component masses. If these systems have accurately determined ages, they act as benchmarks for understanding the evolution of fundamental stellar parameters and allow the calibration of widely used stellar evolution models.

One such system in the solar neighborhood is NO UMa (HIP 61100, HD 109011, GJ 1160). NO UMa is a pair of K-type dwarfs at a distance of $d=25.93 \pm 0.02 \mathrm{pc}$ (see Section 6). The system was observed during the CORAVEL radial velocity (RV) survey (Baranne et al. 1979; Duquennoy et al. 1991) where its spectroscopic binarity was discovered. At the time, no CORAVEL RV curve or spectroscopic orbit parameters for NO UMa were presented in the literature, although the statistical studies of K-type binaries in Mayor 
et al. (1992) and Halbwachs et al. (2000) from the CORAVEL survey presumably included the system. Arenou et al. (2000) presented the first orbit solution, including preliminary component masses, using the CORAVEL RV data and Intermediate Astrometric Data from Hipparcos catalog (Perryman et al. 1997).

Further follow-up by Strassmeier et al. (2000) revealed chromospheric activity and $\mathrm{Li}$ absorption, indications of a relatively young age. The first spectroscopic orbit parameters were provided by Halbwachs et al. (2003) and an independent astrometric orbit from the Hipparcos data was presented in Goldin \& Makarov (2007). In a subsequent paper, Strassmeier et al. (2012) presented updated RV curves for both components of the binary, provided a spectroscopic orbit solution, and updated fundamental and spectroscopic parameters of each component. The orbit parameters estimated in these studies and the Hipparcos distance indicated that the components of the NO UMa system may be resolvable at angular separations $\lesssim 0$ "' 1 ; feasible with modern adaptive optics (AO) systems on large aperture telescopes. Additionally, King et al. (2003) proposed the star as a nuclear member of the Ursa Major moving group (UMaG) (or cluster), a $\sim 500 \mathrm{Myr}$ old group of coeval stars with common Galactic kinematics.

Thus, NO UMa is an attractive target for high angular resolution, high-contrast AO imaging, not only to resolve the binary components, but also to search for low-mass companions. Stellar binaries are typically excluded from exoplanet imaging surveys, however, NO UMa's component separation is small enough ( $\sim 2-3 \mathrm{AU})$ that circumbinary companions on wide orbits are not dynamically unstable (Thalmann et al. 2014, and references therein). Although challenging for planet formation theory (Kley \& Haghighipour 2014), recent work has revealed circumbinary disks with the potential to form planets (e.g., Dutrey et al. 2014; Tang et al. 2014; Rapson et al. 2015), a few binaries with directly imaged, circumbinary, planet/brown dwarf companions (Delorme et al. 2013; Kraus et al. 2014), and numerous circumbinary planets in transit (Welsh et al. 2015, and references therein). For these reasons, we included NO UMa as a target in the LEECH exoplanet imaging survey (Skemer et al. 2014a) and succeeded in resolving the individual components.

In this work, we describe the derivation of a full set of orbital parameters for NO UMa to provide component masses, estimate the fundamental parameters of each component using our resolved photometry, revise the system's Galactic kinematics using new measurements from our orbit fit, and compare our measured masses to model predictions. In Section 2 we summarize the LEECH program and NO UMa's inclusion as a target. In Section 3 we provide details on the available fundamental properties and orbital parameters of NO UMa. Section 4 describes our AO imaging of the target, an archival imaging data set, and the data reduction. In Section 5 we describe the imaging analyses and results. We combine our astrometric measurements with existing data to solve the complete orbit of the binary and improve component mass constraints by a factor $\gtrsim 6$ in Section 6 . We present in Section 7 fundamental parameters and revised kinematics of the binary components and compare the estimated component masses to those predicted by theoretical evolution models. Section 8 provides a summary.

\section{THE LBT INTERFEROMETER (LBTI) EXOZODI EXOPLANET COMMON HUNT (LEECH) EXOPLANET IMAGING SURVEY}

The LEECH is a multi-national collaboration using the Large Binocular Telescope (LBT) coupled with the dual deformable secondary LBT AO system (FLAO, LBTIAO, Esposito et al. 2010, 2011; Riccardi et al. 2010; Bailey et al. 2014). LEECH uses the $L / M$-band Infrared Camera (LMIRcam, Skrutskie et al. 2010; Leisenring et al. 2012) of the LBTI (Hinz et al. 2008) to conduct the first large-scale exoplanet imaging survey at thermal infrared (IR) wavelengths ( $L^{\prime}$-band, $\lambda_{c} \approx 3.8 \mu \mathrm{m}$ ) over $\sim 100$ nights (Skemer et al. 2014a). LEECH takes advantage of two key features of searching for planets in the thermal IR. First, because of strong molecular absorption at shorter wavelengths, giant exoplanet fluxes peak between $\sim 4-5 \mu \mathrm{m}$ (Burrows et al. 1997). Second, AO systems perform better at longer wavelengths and provide optimal correction (Beckers 1993). Therefore, the LEECH survey is sensitive to older, cooler planets $(\lesssim 1 \mathrm{Gyr}, \lesssim 1000 \mathrm{~K})$ and complements other next generation exoplanet surveys searching for younger $(\lesssim 200 \mathrm{Myr})$, hotter planets in the near-IR (GPI, SPHERE, Project 1640, Beuzit et al. 2008; Macintosh et al. 2008; Hinkley et al. 2011).

Targets in the LEECH survey span the relatively unexplored age range of $\sim 0.1-1 \mathrm{Gyr}$, a range where LBTI/LMIRcam remains sensitive to both "hot-start" and "cold-start" planets (Spiegel \& Burrows 2012; Marleau \& Cumming 2014). The targets are drawn from several samples that include nearby Aand B-type stars, very nearby, $\leqslant 1$ Gyr old FGK stars, and more than 50 stars in the intermediate-age UMaG. NO UMa is included as a LEECH target in this subsample. The sensitivity and utility of the LBTAO coupled with LBTI/LMIRcam has been demonstrated in several studies of known, substellar companions and a very low-mass binary (Skemer et al. 2012, 2014b; Bonnefoy et al. 2014; Schlieder et al. 2014). These capabilities also led to strong constraints on the possibility of a fifth planet in the HR 8799 planetary system during the LEECH survey (Maire et al. 2015). Further technical details of the LEECH survey, including $H$ and $L^{\prime}$ contrast curves, are provided in Skemer et al. (2014a).

\section{KNOWN FUNDAMENTAL AND ORBITAL PROPERTIES OF NO UMA}

NO UMa was identified decades ago as a K2V standard in the Morgan-Keenan (MK) system via visual inspection of photographic spectrograms (Johnson \& Morgan 1953). This spectral type (SpTy) is the integrated type for both components and has changed very little since first proposed. Independent determinations in the literature range from $\sim \mathrm{K} 1 \mathrm{~V}$ to $\mathrm{K} 3 \mathrm{~V}$ (e.g., Yoss 1961; Heinze \& Hinz 2005). Using available optical photometry for the NO UMa system (Table 1) and the mainsequence color-temperature conversions of Pecaut \& Mamajek $(2013)^{15}$, we interpolate a median SpTy of K2.5 \pm 0.5 using Monte Carlo (MC) methods. We therefore conservatively adopt an integrated system SpTy of K2Ve \pm 1 (" $e$ " for emission, see below).

Arenou et al. (2000) used RV data from a CORAVEL survey of late-type, main-sequence stars and Intermediate Astrometric

\footnotetext{
15 Throughout this work, we use the expanded table available on Eric Mamajek's webpage: http://www.pas.rochester.edu/ emamajek/EEM_ dwarf_UBVIJHK_colors_Teff.txt
} 
Table 1

Summary of NO UMa Physical Properties

\begin{tabular}{|c|c|c|c|c|}
\hline & NO UMa & NO UMa A & NO UMa B & References \\
\hline$\alpha_{J 2000}\left(^{\circ}\right)$ & 187.828876 & $\ldots$ & $\ldots$ & (1) \\
\hline$\delta_{J 2000}\left({ }^{\circ}\right)$ & +55.118858 & $\ldots$ & $\ldots$ & (1) \\
\hline 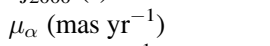 & $107.08 \pm 1.20$ & $\ldots$ & $\ldots$ & (2) \\
\hline $\mathrm{d}(\mathrm{pc})$ & $25.87 \pm 0.02$ & $\ldots$ & $\ldots$ & (8) \\
\hline systemic RV $\left(\mathrm{km} \mathrm{s}^{-1}\right)$ & $-9.873 \pm 0.007$ & $\ldots$ & $\ldots$ & (8) \\
\hline$v \sin i\left(\mathrm{~km} \mathrm{~s}^{-1}\right)$ & $\ldots$ & $5 \pm 1$ & $6 \pm 1$ & (3) \\
\hline$V(\mathrm{mag})$ & $8.13 \pm 0.03$ & $\ldots$ & $\ldots$ & (4) \\
\hline$B_{T}(\mathrm{mag})$ & $9.29 \pm 0.02$ & $\ldots$ & $\ldots$ & (5) \\
\hline$V_{T}(\mathrm{mag})$ & $8.21 \pm 0.01$ & $\ldots$ & $\ldots$ & (5) \\
\hline$J(\mathrm{mag})$ & $6.32 \pm 0.03$ & $\ldots$ & $\ldots$ & (1) \\
\hline$H$ (mag) & $5.81 \pm 0.03$ & $6.27 \pm 0.10$ & $6.96 \pm 0.12$ & $(1),(8)$ \\
\hline$K_{s}(\mathrm{mag})$ & $5.66 \pm 0.02$ & $6.12 \pm 0.03$ & $6.83 \pm 0.04$ & (1), (8) \\
\hline Mass $\left(M_{\odot}\right)$ & $1.47 \pm 0.03$ & $0.83 \pm 0.02$ & $0.64 \pm 0.02$ & (8) \\
\hline Age (Myr) & $500 \pm 100$ & $\ldots$ & $\ldots$ & (7) \\
\hline
\end{tabular}

References. (1) Cutri et al. (2003), (2) Arenou et al. (2000), (3) Strassmeier et al. (2012), (4) Mermilliod \& Mermilliod (1994), (5) Høg et al. (2000), (6) Cutri (2013); Wright et al. (2010), (7) King et al. (2003); Brandt \& Huang (2015), (8) This work.

Data from the Hipparcos mission to estimate orbital parameters for the NO UMa system. The semimajor axis of the Hipparcos photocenter was combined with the period, eccentricity, mass ratio, and other parameters from the CORAVEL RV curve to place constraints on individual component masses. They estimate the primary and secondary masses with relative errors of $\sim 25 \%$ and $\sim 17 \%$, respectively. Their analysis also provided a revised parallax and proper motions that were corrected for the motion of the Hipparcos photocenter.

Strassmeier et al. (2000) obtained high-resolution optical spectroscopy and photometric monitoring of NO UMa in their search for late-type Doppler-imaging targets. Their Kitt Peak National Observatory $0.9 \mathrm{~m}$ coudé feed spectra revealed Ca II $\mathrm{H}$ \& $\mathrm{K}$ chromospheric emission and weak $\mathrm{Li}$ absorption in the system with a $34 \pm 7 \mathrm{~m} \AA$ equivalent width. Their Strömgren $y$ photometry from the $0.75 \mathrm{~m}$ Vienna Observatory automatic photometric telescope (APT) "Wolfgang" provided an estimated period of $\sim 8.3$ days. The observed activity, $\mathrm{Li}$, and rotation are indicative of an age $\lesssim 625 \mathrm{Myr}$ but $>125 \mathrm{Myr}$ in a \% 2-type star (Mamajek \& Hillenbrand 2008; King \& Schuler 2005).

Following their initial study, Strassmeier et al. (2012) present dedicated spectroscopic monitoring of NO UMa using the $1.2 \mathrm{~m}$ STELLA-I telescope and the STELLA Echelle Spectrograph (SES) on Tenerife. Their SES data consisted of 129 spectra obtained over 1629 days. They measured individual component velocities in each spectrum to generate RV curves and solve the spectroscopic orbit. Their high-quality spectra covering the entire orbit allow them to derive a period, time of periastron, eccentricity, and systemic RV to $\lesssim 1 \%$ precision. They also reanalyze their APT photometric data that consists of 60 observations over 135 days to obtain a new photometric period of $8.4 \pm 0.2$ days. They attribute this period to rotational modulation of the primary. Considering the primary's approximately early-K SpTy, this is broadly consistent with its $5 \pm 1 \mathrm{~km} \mathrm{~s}^{-1} v$ sini. Strassmeier et al. (2012) also derive fundamental and spectroscopic parameters for each component using the synthetic spectrum fitting package PARSES (Allende Prieto 2004; Jovanovic et al. 2013). These include effective temperatures of $5030 \pm 75$ and $4900 \pm 150 \mathrm{~K}$ for the primary and secondary, respectively. We also note that orbital parameters from the CORAVEL RV data are briefly discussed in Halbwachs et al. (2003) and an independent estimate of the orbit from the Hipparcos intermediate data is presented in Goldin \& Makarov (2007).

Balega et al. (2013) also present speckle interferometry measurements of NO UMa from the $6 \mathrm{~m} \mathrm{BTA}$ telescope at the Special Astrophysical Observatory of the Russian Academy of Sciences (SAO-RAS). Their observations span $\sim 4$ years from 2002 to 2006 and were obtained using filters with $\lambda_{c}=545$, 750 , or $800 \mathrm{~nm}$. We describe their data in more detail in Section 6 .

\section{OBSERVATIONS AND DATA REDUCTION}

\subsection{LEECH LBTI/LMIRcam Imaging}

NO UMa was observed using LBTI/LMIRcam during two LEECH observing runs in 2013. The LBTI is located at the bent Gregorian focus of the LBT and does not have a derotator. Only the right side of the LBT was used during the observations (the "DX" side). The LBT AO system was driven using NO UMa as a natural guide star.

On 2013 April 22 UT we obtained $200 \times 0.495 \mathrm{~s}$ exposures of NO UMa with the $L^{\prime}$-band filter $\left(\lambda_{c}=3.70 \mu \mathrm{m}\right.$, $\Delta \lambda=0.58 \mu \mathrm{m})$. The binary was dithered to two positions in the field of view separated by $4 . " 5$. We also observed a star with similar SpTy, HIP 46580 (K3V), immediately after NO UMa to calibrate the telescope+detector point-spread function 

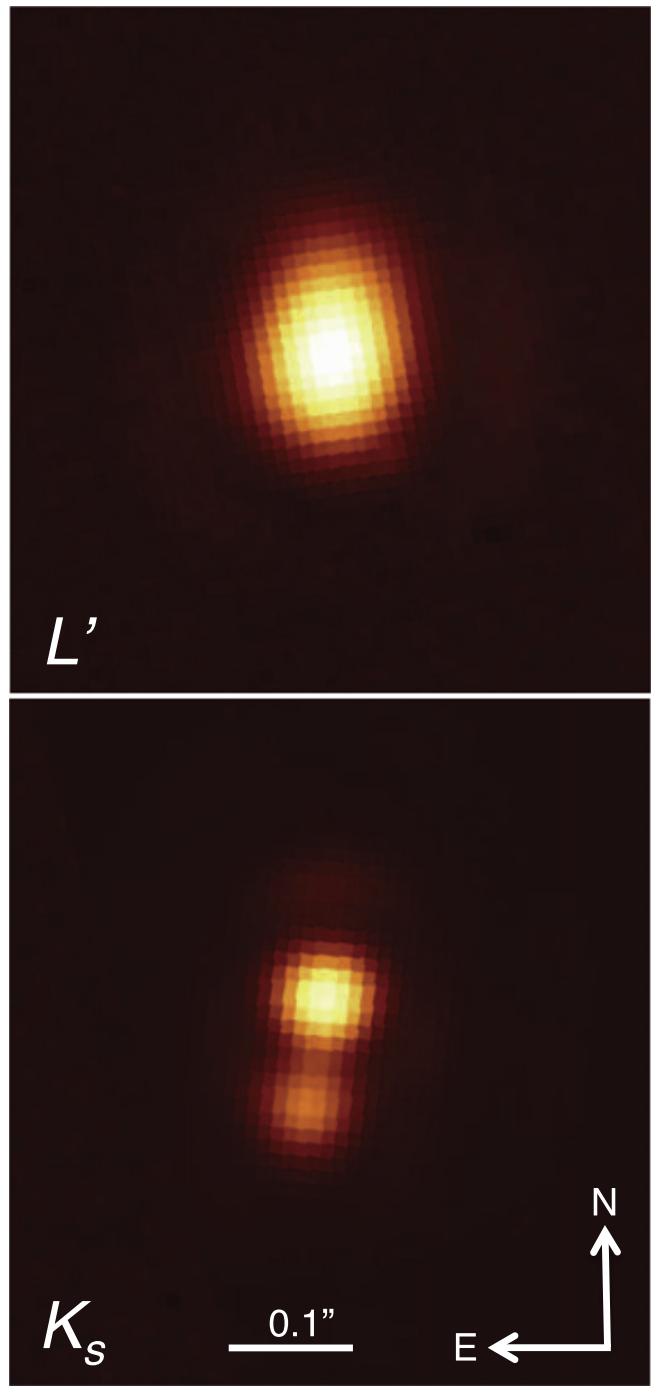

Figure 1. LBTI/LMIRcam $L^{\prime}$-band (top) and $K_{s}$-band (bottom) images of NO UMa A and B. The $L^{\prime}$ image was obtained on 2013 April 22 UT and the $K_{s}$ image was obtained on 2013 December 26 UT. The binary components are blended at $L^{\prime}$ but well resolved at $K_{s}$. Our analyses estimate an $L^{\prime}$ separation of $64.7 \pm 0.2$ mas and a $K_{s}$ separation of $86.2 \pm 0.4$ mas. The system exhibits $\sim 180^{\circ}$ of position angle change and $\sim 20$ mas of separation change in only 7 months.

(PSF). Our reduction includes corrections for distortion effects, detector bias, sky background, and bad pixels followed by frame re-centering via cross-correlation and averaging. The blended components exhibit an elongated intensity distribution in the $L^{\prime}$ images (Figure 1). We obtained second epoch LBTI/ LMIRcam images of NO UMa on December 26 UT 2013 in the $H\left(\lambda_{c}=1.65 \mu \mathrm{m}, \Delta \lambda=0.31 \mu \mathrm{m}\right)$ and $K_{s}$-band filters $\left(\lambda_{c}=2.16 \mu \mathrm{m}, \Delta \lambda=0.32 \mu \mathrm{m}\right)$. The $H$ - and $K_{s}$-band observations each consisted of $100 \times 0.058 \mathrm{~s}$ exposures dithered to two positions separated by $4 . \prime 5$. We followed the same reduction steps for the $H$ and $K_{s}$ frames as for the $L^{\prime}$ frames. The components are well resolved in both the $H$ and $K_{s}$ bands. The $K_{s}$ image is shown in Figure 1. We observed a photometric calibrator immediately after the observations in both near-IR bands, but the PSF of the calibrator was not useful for subsequent analyses due to an issue with the AO that affected only the calibrator observations (see Section 5).

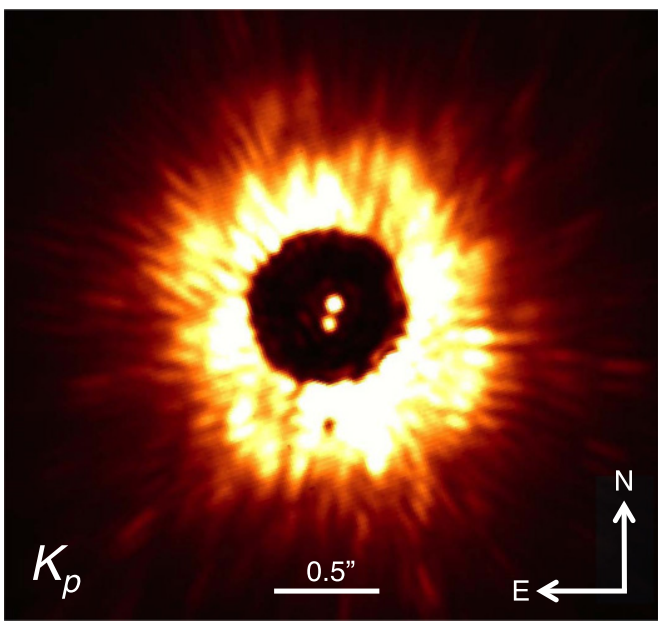

Figure 2. Keck/NIRC2 $K_{p}$-band image of NO UMa A and B. The image was obtained on 2010 May 27 UT. The binary is clearly resolved behind the 0.6 translucent mask. The components are separated by $77.7 \pm 0.7$ mas. The position angle of the secondary in this earlier epoch is very close to our 2013 December UT LBTI/LMIRcam images. The bright halo surrounding the mask is scattered light.

\subsection{Keck II/NIRC2 Archival Imaging}

NO UMa was observed on May 272010 UT using Keck II/ Near Infrared Camera 2 (NIRC2) coupled with NGS $\mathrm{AO}^{16}$ (Wizinowich et al. 2000). The data were obtained in the $K_{p}$ filter $\left(\lambda_{c}=2.124 \mu \mathrm{m}, \Delta \lambda=0.351 \mu \mathrm{m}\right)$ with the narrow camera setting yielding a field-of-view of 10 !' $2 \times 10$ " 2 . Eight frames were obtained with NO UMa placed behind the 0" 6 diameter translucent focal plane mask. Three of these images had exposure times of $5.0 \mathrm{~s}$ and the remaining had exposure times of $60.0 \mathrm{~s}$. The binary is clearly resolved behind the mask in all eight frames (Figure 2). Saturated images of NO UMa were also obtained at four different dither locations to estimate the sky background. The NIRC2 data reduction included cosmic ray and bad pixel removal, dark subtraction, flat fielding, and sky subtraction. Optical distortions were corrected using the NIRC2 distortion solution provided by the Keck observatory.

\section{IMAGE ANALYSES}

Since the binary is blended in our $L^{\prime}$ images, the component separation and flux ratio were calculated by fitting the data with a two-star model constructed from the PSF of the nearby standard using Levenberg-Marquardt minimization. We find the components have $\Delta L^{\prime}=0.73 \pm 0.01$ and are separated by $6.046 \pm 0.014$ pixels. We converted the measured separation in pixels to mas using the LMIRcam plate scale of $10.707 \pm 0.012$ mas pix $^{-1}$ from Maire et al. (2015) to arrive at an angular separation of $64.7 \pm 0.2$ mas. We calculate the magnitude of the primary using $m_{1}=$ $m_{12}+2.5 \cdot \log _{10}\left(1+10^{-\Delta m / 2.5}\right)$, where $m_{1}$ is the primary magnitude, $m_{12}$ is the unresolved, integrated magnitude of both components, and $\Delta m$ is the measured magnitude difference. Since no calibrated $L^{\prime}$ photometry is available for NO UMa, we estimate the component photometry using the WISE W1 magnitude of the system as an approximation for $m_{12}$ in the previous equation. The secondary magnitude, $m_{2}$, is then

${ }^{16}$ Program ID K319N2, PI Armandoff. 
calculated from $\Delta L^{\prime}$ and $m_{1}$. The approximate $L^{\prime}$-band system photometry is provided in Table 1 .

We detected no additional companions in the LMIRcam field of view. Since the binary was partially resolved in our $L^{\prime}$ images, we did not perform a full deep imaging sequence to search for planetary mass companions. However, our short integrations were sensitive to circumbinary tertiary companions with $\Delta L^{\prime}=5 \mathrm{mag}$ at separations $>0{ }^{\prime \prime} 5$. At the $\sim 500 \mathrm{Myr}$ age of the system (see Section 7.2), this magnitude ratio and angular separation correspond to companion masses $M \gtrsim 0.1 M_{\odot}$ (Baraffe et al. 1998) at projected separations $\gtrsim 13 \mathrm{AU}$.

Issues with the PSF calibrator in the $H$ and $K_{s}$ bands did not permit analysis of those images using the same fitting routine employed for the $L^{\prime}$ data. We modified the procedure to allow the PSF to vary as an additional free parameter in the fit minimization. The modified procedure resulted in a best-fit magnitude difference and pixel separation in the $K_{s}$ band of $\Delta K_{s}=0.71 \pm 0.02 \mathrm{mag}$ and $8.051 \pm 0.019$ pixels, respectively. In the $H$ band, the new method provides $\Delta H=0.69 \pm 0.06$ and a separation of $8.02 \pm 0.16$ pixels. The larger uncertainties in the $H$ band are a result of a poorer fit and larger residuals in the minimization. We conservatively adopt flux ratio and separation errors of $5 \%$ and $2 \%$ in this band. We used the LMIRcam plate scale to calculate an $H$-band angular separation of $85.9 \pm 1.7$ mas. When calculating the $K_{s^{-}}$ band separation in the same way, we find that it does not overlap with the $H$-band separation within $1 \sigma$ uncertainties. To compensate, we add the difference between the nominal $H$ and $K_{s}$ separations in quadrature to the measured $K_{s}$ uncertainty as an extra systematic error to arrive at a final $K_{s}$ angular separation of $86.2 \pm 0.4$ mas. Following the same procedure described for the $L^{\prime}$ data, we used the unresolved Two Micron All-Sky Survey (2MASS, Cutri et al. 2003) $H$ - and $K_{s}$-band photometry and the measured magnitude differences to calculate the $H$ and $K_{s}$ component photometry. These near-IR magnitudes are listed in Table 1.

Since LMIRcam has no derotator, the $L^{\prime}, H$, and $K_{s}$ images must be re-oriented with true North. We corrected the measured position angles from our binary fitting routine using the detector orientation of $-0^{\circ} .430 \pm 0.076$ east of north derived from images of the $\Theta^{1}$ Ori $C$ field in Maire et al. (2015). Each reduced, combined image was also corrected for the median parallactic angle during the image sequence to align with sky coordinates. We adopt position angle errors for the final images that reflect the full range of parallactic angles during each image sequence (a maximum of 1.8 for the $H$ and $K_{s}$ observations, see Table 3 ).

We do not use the $K_{p}$-band photometry from the archival Keck/NIRC2 images due to the use of the translucent coronagraphic mask in those observations. We measured the angular separation and position angle of the secondary in each of the eight reduced images from their DS9 WCS coordinates. The mean and standard deviation of each parameter was calculated and each are provided in Table 3.

\section{ORBIT ANALYSIS}

To determine the orbit of NO UMa, we follow standard binary orbit formalism (see the Appendix) using the methods presented in Esposito et al. (2013). In short, initial guesses of $P$, $T_{0}, e, K_{1}, K_{2}$, and $\gamma$ are made that are compatible with the observed data and correlations between orbital parameters.
Then, a simultaneous astrometric and spectroscopic best-fit orbit solution is solved using Levenberg-Marquardt leastsquares minimization. We tested ranges of initial guesses to investigate the effect on the resulting fit parameters. Due to the good coverage of both our astrometric and spectroscopic data over different phases of the orbit, we found that choices of initial guess comparable to previous estimates have no significant effect on the results of the fits. The measured $t, x$, and $y$ inputs to the orbit analysis include those from our three LBTI/LMIRcam images, the Keck/NIRC2 image, and the six speckle interferometry measurements from Balega et al. (2013). ${ }^{17}$ The measured astrometry is provided in Table 3 . The measured $v_{1}$ and $v_{2}$ values used in the fit are the STELLAI/SES observations from Strassmeier et al. (2012) described in Section 3. When exploring this RV data, we found that in a spectroscopic only fit, the measured RV errors resulted in correlated fit residuals for the primary and secondary that had standard deviations of $\sim 0.1$ and $\sim 0.2 \mathrm{~km} \mathrm{~s}^{-1}$, respectively. These SES systematics were previously investigated by Weber \& Strassmeier (2011, see their Figure 1) and are likely instrumental or calibration effects. To compensate for this underestimation of the true uncertainty, we added in quadrature the standard deviation of the residuals from each of the fits back into the measured errors of each component as an additional error term.

We present our best-fit orbital elements in the first column of Table 2 in comparison to the previous best estimates. The $1 \sigma$ uncertainties in the elements were estimated using MC methods where we drew $10^{3}$ random trials of the astrometric measurements from Gaussian error distributions around the nominal values and repeated the minimization procedure. Our independent estimates of the orbital parameters are consistent within $3 \sigma$ with those previously reported using both astrometric and spectroscopic data. Our constraints match well with those derived by Arenou et al. (2000) and Strassmeier et al. (2012). Our more precise astrometric measurements allow us to place much tighter constraints on each of the orbital elements when compared to the Arenou et al. (2000) solution. Figure 3 shows our best-fit orbit compared to the observed astrometric data.

We used our best-fit orbital elements to derive the NO UMa component masses following Equations (11) and (12). Propagating the associated uncertainties, we calculate $M_{1}=0.83 \pm 0.02 M_{\odot}$ and $M_{2}=0.64 \pm 0.02 M_{\odot}$, respectively. We then combined the masses to find $M_{\text {tot }}=1.47 \pm$ $0.03 M_{\odot}$. Our primary and secondary mass estimates are consistent with those of previous studies but with much smaller relative errors of $\sim 2 \%$ and $\sim 3 \%$, respectively. We combined the inclination from our fit with the RV only mass constraints from Strassmeier et al. (2012) and found system and component masses in agreement with our estimates at better than $1 \sigma$. We also derived a new distance to the system, $d=25.87 \pm 0.02 \mathrm{pc}$, slightly farther than the Hipparcos measurement of $25.10 \pm 0.67 \mathrm{pc}$ (van Leeuwen 2007), but consistent within $\sim 1.2 \sigma$.

\section{DISCUSSION}

\subsection{Physical Properties of NO UMa A and B}

To estimate the physical properties of NO UMa A and B, we use our measured values of $K_{s}$ and distance and $M_{*}$ and

\footnotetext{
17 The position angles of five of the six speckle interferometry points were rotated by $180^{\circ}$ to converge on an orbital solution (see Table 3 ).
} 
Table 2

Summary of NO UMa Orbital Properties

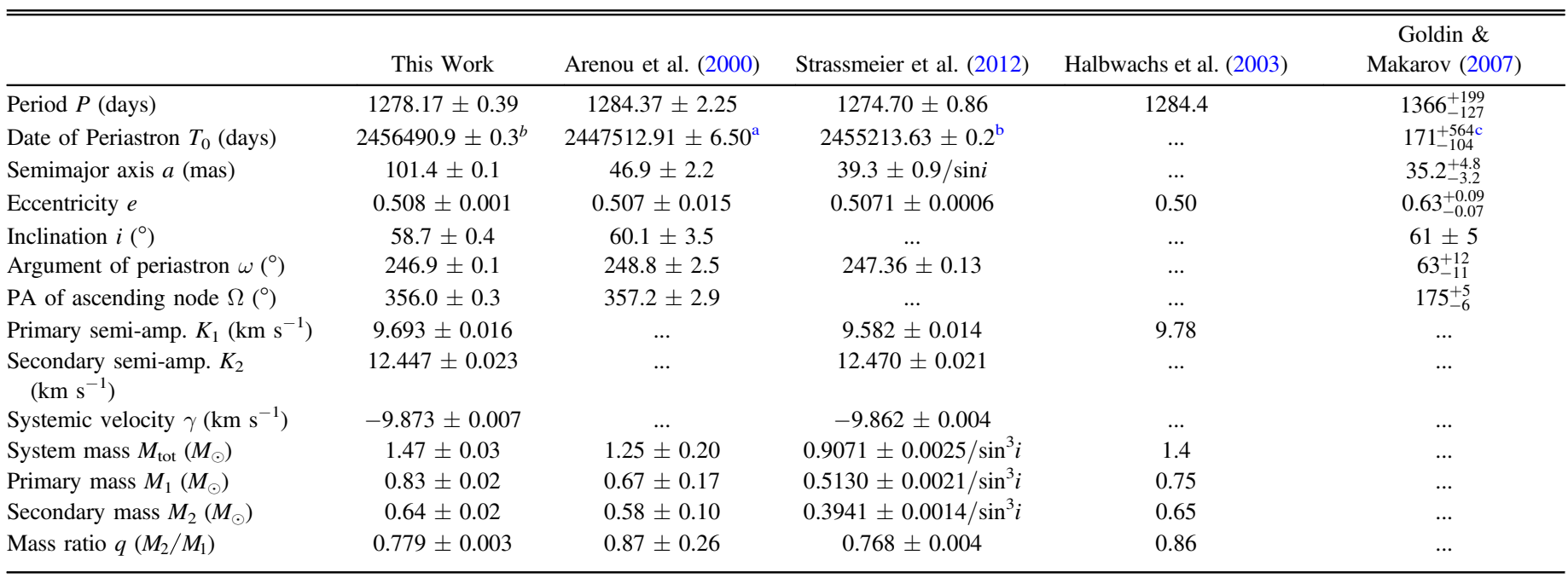

Notes.

a Julian Date.

${ }^{\mathrm{b}}$ Heliocentric Julian Date.

${ }^{\mathrm{c}}$ Days after phase $=0$.

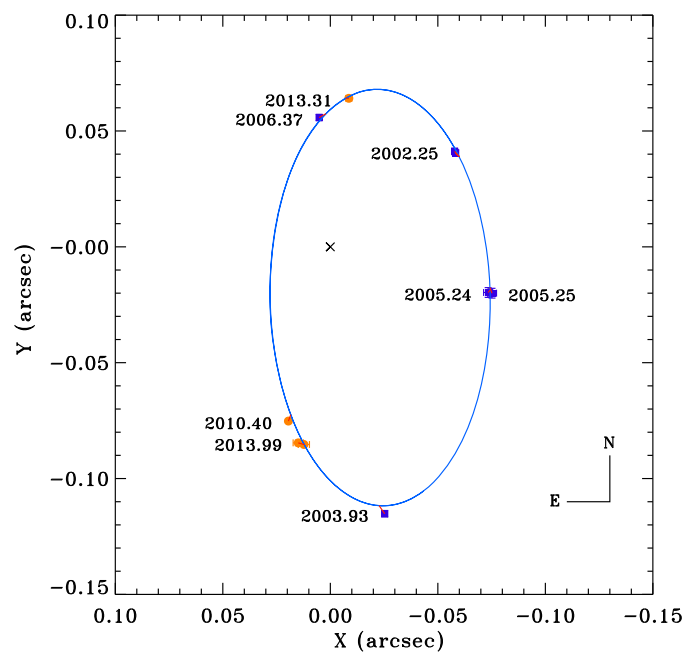

Figure 3. Best-fit orbit of NO UMa B (blue ellipse) with respect to NO UMa A (cross). The fit is compared to the relative astrometry from $\mathrm{AO}$ imaging (orange circles) and speckle interferometry (blue squares) measurements. Short red lines connect the data points to their expected positions along the orbit on the dates of observation. The $X$ and $Y$ coordinates correspond to R.A. and decl., respectively. The orbit is counter clockwise with a period of $\sim 3.5$ years. The error bars of some of the measurements are within the plot symbols.

interpolate within the main-sequence color-temperature conversion table of Pecaut \& Mamajek (2013). To estimate the errors on our interpolated values, we employ MC techniques assuming Gaussian error distributions. The final values and their errors are the medians and dispersions of the interpolated distributions.

We combine the measured $K_{s}$ magnitude of NO UMa A and the distance to the system to find an absolute $K_{s}$-band magnitude $M_{K_{s}}=4.06 \pm 0.03$. We use this measurement to estimate a SpTy and effective temperature of $\mathrm{K} 2.0 \mathrm{~V} \pm 0.5$ and $T_{\text {eff }}=5010 \pm 50 \mathrm{~K}$, respectively. For NO UMa B, we estimate a SpTy of $\mathrm{K} 6.5 \mathrm{~V} \pm 0.5$ and $T_{\text {eff }}=4140 \pm 30 \mathrm{~K}$ from
Table 3

Astrometry for NO UMa

\begin{tabular}{lcccc}
\hline \hline $\begin{array}{l}\text { UT Date } \\
\text { (YYYY MM DD) }\end{array}$ & Band & $\begin{array}{c}\rho \\
(\mathrm{mas})\end{array}$ & $\begin{array}{c}\text { PA } \\
\left({ }^{\circ}\right)\end{array}$ & $\begin{array}{c}\text { O-C }{ }_{X, Y} \\
(\mathrm{mas})\end{array}$ \\
\hline \multicolumn{5}{c}{$6 \mathrm{~m} \mathrm{BTA} /$ Speckle Interferometry } \\
\hline 2002 Apr 03 & $\lambda 545^{\mathrm{a}}$ & $71 \pm 2$ & $305.5 \pm 1.0^{\mathrm{b}}$ & $2.1,2.3$ \\
2002 Apr 03 & $\lambda 750^{\mathrm{a}}$ & $71 \pm 2$ & $304.8 \pm 1.4^{\mathrm{b}}$ & $1.6,1.6$ \\
2003 Dec 04 & $\lambda 800^{\mathrm{a}}$ & $118 \pm 2$ & $192.4 \pm 1.4^{\mathrm{b}}$ & $-2.6,-3.5$ \\
2005 Dec 27 & $\lambda 545^{\mathrm{a}}$ & $76 \pm 3$ & $255.0 \pm 1.7^{\mathrm{b}}$ & $0.9,-1.0$ \\
2005 Mar 31 & $\lambda 800^{\mathrm{a}}$ & $78 \pm 3$ & $254.5 \pm 2.1^{\mathrm{b}}$ & $-1.0,-2.5$ \\
2006 May 17 & $\lambda 545^{\mathrm{a}}$ & $56 \pm 2$ & $5.3 \pm 1.4$ & $3.1,-1.7$ \\
\hline \multicolumn{5}{c}{ Keck/NIRC2 } \\
\hline 27/05/2010 & $K_{p}$ & $77.7 \pm 0.7$ & $165.5 \pm 0.3$ & $1.1,-2.8$ \\
\hline \multicolumn{5}{c}{ LBT/LMIRcam } \\
\hline 22/04/2013 & $L^{\prime}$ & $64.7 \pm 0.2$ & $352.4 \pm 1.3$ & $-1.4,-0.1$ \\
$26 / 12 / 2013$ & $H$ & $85.9 \pm 1.7$ & $170.0 \pm 1.8$ & $2.8,0.7$ \\
$26 / 12 / 2013$ & $K_{s}$ & $86.2 \pm 0.4$ & $171.8 \pm 1.8$ & $0.2,0.1$ \\
\hline
\end{tabular}

Notes.

${ }^{\mathrm{a}}$ Central wavelength in $\mathrm{nm}$ of the filter used during the observation.

${ }^{b}$ PA measurement from Balega et al. (2013) rotated by $180^{\circ}$ to be consistent with previously estimated orbit parameters.

$M_{K_{\mathrm{s}}}=4.77 \pm 0.04$. Using the measured masses of the A and $\mathrm{B}$ components, we estimate parameters in the same way and find values that are consistent with those estimated from $M_{K_{s}}$ but with larger uncertainties. The SpTy and effective temperature estimates using $M_{K_{s}}$ and the masses are also consistent with the integrated SpTy of the system. Using the more precise $M_{K_{s}}$ temperature estimates, our $T_{\text {eff }}$ of NO UMa A is consistent with the previous estimate from Strassmeier et al. (2012) but our $T_{\text {eff }}$ for the B component is $\sim 750 \mathrm{~K}$ cooler. This can likely be attributed to their use of a blended spectrum to 
estimate the temperature of each component rather than resolved measurements.

We also used our measured $M_{K_{s}}$ values to estimate component luminosities. Interpolating from the Pecaut \& Mamajek (2013) table, we estimate $\log \left(L / L_{\odot}\right)=-0.49 \pm 0.03$ for NO UMa A and $\log \left(L / L_{\odot}\right)=-0.97 \pm 0.02$ for NO UMa B. These luminosities and the other physical parameters of the components estimated using $M_{K_{s}}$ are compiled in Table 1.

\subsection{NO UMa and the UMaG}

References to a group of stars sharing similar kinematics in and around the constellation Ursa Major date back nearly 150 years (e.g., Proctor 1869). A full history of the associated literature is beyond the scope of this paper but we do summarize some of the modern studies of the UMaG and evaluate NO UMa's membership in it using our new data.

A detailed, early study of the UMaG was presented by Roman (1949) who investigated the kinematics of all proposed members at that time. We point the interested reader to her exhaustive summary of previous works related to the group. Roman's analysis revealed a compact nucleus of 14 stars surrounded by a larger stream extending to radii of $\sim 100 \mathrm{pc}$. This early list already included NO UMa (listed as HD 109011) as a nuclear member of the group. The work of Soderblom \& Mayor (1993) used chromospheric activity (as traced by Ca II $\mathrm{H}$ $\& \mathrm{~K})$ and improved kinematic measurements to identify a list of $43 \mathrm{UMaG}$ members.

The most recent comprehensive studies of the group include those of King et al. (2003), King \& Schuler (2005), and Ammler-von Eiff \& Guenther (2009). King et al. (2003) sought to reinvestigate previously proposed members with new astrometric, photometric, and spectroscopic data. From an input list of $\sim 220$ proposed UMaG candidates, they identify 57 probable and possible members that are well defined in kinematic and color-magnitude space. Their results confirmed NO UMa as a bona-fide member of the UMaG nucleus. Comparison of evolution models to the empirical colormagnitude diagram (CMD) of their refined membership lists suggested an age of $500 \pm 100 \mathrm{Myr}$ for the group. The followup paper of King \& Schuler (2005) examined activity, Li depletion, and abundances in members and affirms that the UMaG has approximately solar metallicity and its age overlaps with the Hyades and Coma Ber clusters within measurement and model uncertainties. Ammler-von Eiff \& Guenther (2009) present a similar study and reach similar conclusions. Tabernero et al. (2014) perform a detailed chemical tagging study of candidate FGK type members of the UMaG and find 29 stars with similar chemical compositions and $[\mathrm{Fe} / \mathrm{H}]=$ $0.03 \pm 0.07$ dex. Using a Bayesian framework, Brandt \& Huang (2015) compared reliable, main-sequence-turn-off, members of the UMaG to modern evolution models that include the effects of stellar rotation and affirmed the $\sim 500$ Myr age of the group. Additional UMaG members are still being proposed, such as the candidate $\mathrm{M}$ dwarf members with measured parallaxes in Shkolnik et al. (2012), Riedel et al. (2014) and Bowler et al. (2015).

Mamajek et al. (2010) present a comprehensive analysis of the UMaG's nuclear membership and kinematics using updated parallaxes from the Hipparcos re-reduction of van Leeuwen (2007). Here we re-evaluate the kinematics of NO UMa using the photocentric motion corrected proper motions from Arenou et al. (2000) and the new systemic RV and distance from our orbit fit. We calculate Cartesian Galactic velocities and positions, UVWXYZ, using the methods outlined by Johnson \& Soderblom (1987) updated for J2000.0 coordinates. ${ }^{18} \mathrm{We}$ calculate $U V W_{\mathrm{NO}} \mathrm{UMa}=(14.19,3.08,-7.70) \pm(0.14,0.07$, $0.14) \mathrm{km} \mathrm{s}^{-1}$ and $X Y Z_{\mathrm{NO} \text { UMa }}=(-7.70,9.51,22.79) \pm(0.01$, $0.01,0.01) \mathrm{pc}$. Our updated calculation of $U V W_{\mathrm{NO}} \mathrm{UMa}$ is a close match to the UMaG nucleus mean velocity of $U V W_{\mathrm{UMaG}}=(15.0,2.8,-8.1) \pm(0.4,0.7,1.0) \mathrm{km} \mathrm{s}^{-1}$ (Mamajek et al. 2010). When compared to the velocities calculated using the Hipparcos proper motions and parallax (van Leeuwen 2007) and the mean system RV from Gontcharov 2006, $U V W_{\text {Hip }}=(15.9,-1.2,-9.9) \pm(0.2,0.3$, $0.4) \mathrm{km} \mathrm{s}^{-1}$, the revised velocities are much improved.

Figure 4 shows projections of the six-dimensional Galactic kinematics of proposed nuclear and stream UMaG members from King et al. (2003) compared to our new estimates of NO UMa's kinematics and those calculated using previously available data. The new kinematic estimates place NO UMa firmly in the tight $U V W$ distribution of the $\mathrm{UMaG}$ nucleus where previously available data placed it as a $\gtrsim 3 \sigma$ outlier in $U$, $V$, and $W$. Mamajek et al. (2010) found that all the kinematic outliers in the UMaG nucleus were proposed binaries and suggested that their larger peculiar velocities may be attributed to binary motion. These results indicate that this hypothesis was true at least in the case of NO UMa. Our revised kinematics and the numerous age indicators described in Section 3 reinforce NO UMa's status as a bona-fide member of the $\mathrm{UMaG}$ nucleus.

\subsection{Model Comparisons}

Both components of the NO UMa system are expected to have settled onto the main sequence at the $500 \mathrm{Myr}$ age of the UMa group and large deviations from model predictions are not expected. However, comparison of our measured masses to model estimates can provide insight into the scatter between the models and reveal any discrepancies between the measurements and model predictions. To explore these possibilities, we use our luminosities to estimate component masses from five sets of stellar evolution models at an age of $500 \mathrm{Myr}$. From the models of Bertelli et al. (2008, Padova models), Dotter et al. (2008, Dartmouth models), Bressan et al. (2012, PARSEC models), Ekström et al. (2012, Geneva models), and Baraffe et al. (2015, BHAC models) with approximately solar abundances, we interpolate the masses and their uncertainties using MC methods and compare directly to our constraints. The model derived masses and model abundances are provided in Table 4.

Figure 5 shows our measured masses compared to the predicted masses from the five sets of models. Each of the model derived masses match our measurements within the $1 \sigma$ uncertainties for each component. There is very little scatter between the masses estimated from each set of models. The small observed scatter can be attributed to differences in abundances and input physics. These comparisons indicate that modern stellar evolution models reproduce well the measured masses of intermediate-age K-type stars.

\footnotetext{
${ }^{18}$ We define $U$ and $X$ positive toward the Galactic center, $V$ and $Y$ positive in the direction of solar motion around the galaxy, and $W$ and $Z$ positive toward the north Galactic pole. In this coordinate system, the Sun lies at the origin.
} 

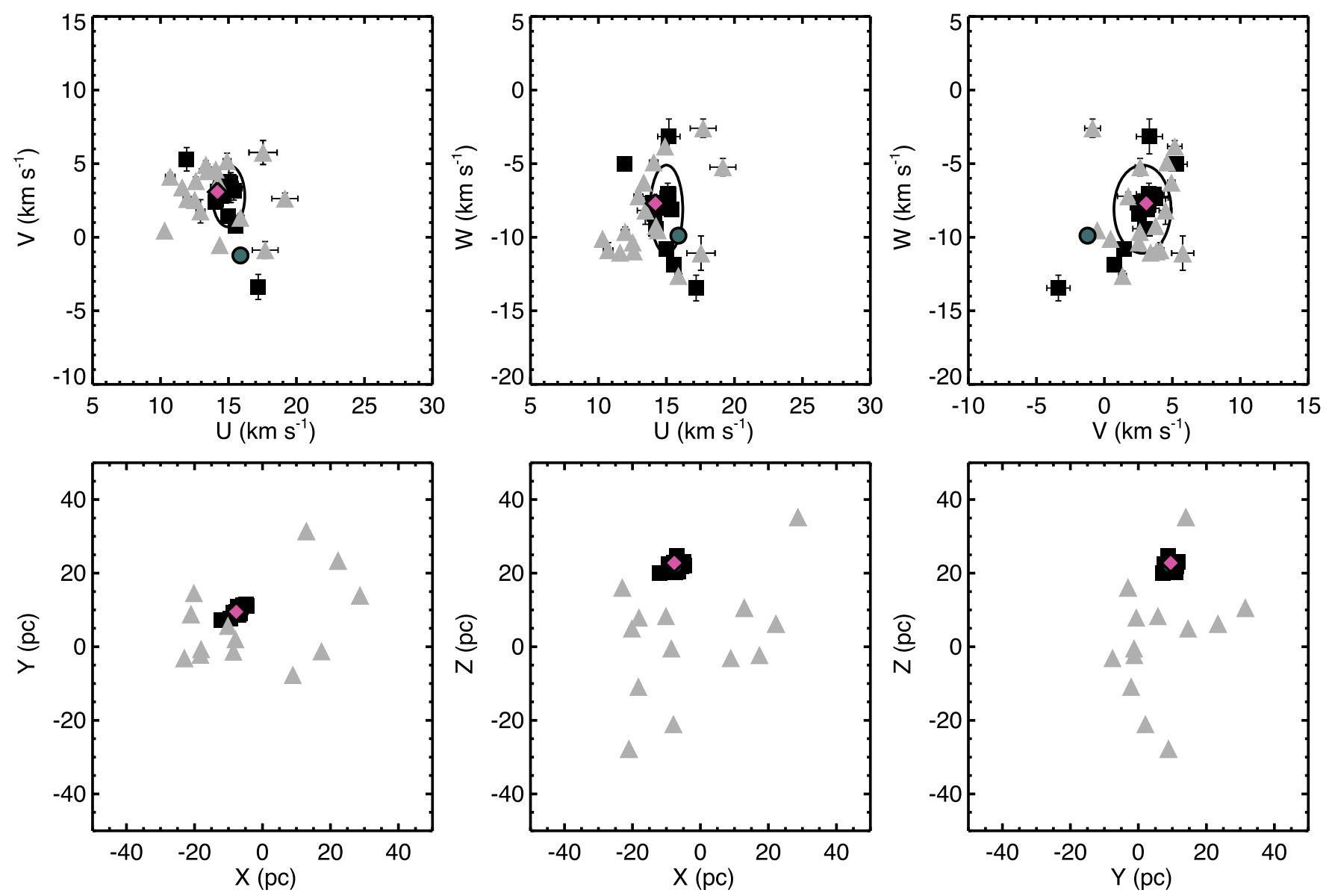

Figure 4. Projections of the six-dimensional Galactic kinematics of the Ursa Major moving group (UMaG) compared to previous (teal circle) and revised (pink diamond) kinematics of NO UMa. Proposed nuclear (black squares) and stream (gray triangles) members are taken from the list of King et al. (2003). (Top) Projections in $U V W$ Galactic velocity. The black ellipse designates the $3 \sigma$ dispersion of the average UMaG nucleus velocities from Mamajek et al. (2010). Our revised NO UMa $U V W$ velocities place it firmly in the UMaG nucleus. (Bottom) Projections of the XYZ Galactic positions. NO UMa and other UMaG nucleus members occupy a very small volume of space, only $\sim 10 \mathrm{pc}^{3}$, and are surrounded by more distant kinematic stream members.

Table 4

Model Component Mass Estimates

\begin{tabular}{lcc}
\hline \hline Model & $\begin{array}{c}\text { Primary Mass } \\
\left(M_{\odot}\right)\end{array}$ & $\begin{array}{c}\text { Secondary Mass } \\
\left(M_{\odot}\right)\end{array}$ \\
\hline $\begin{array}{l}\text { Bertelli et al. (2008) } \\
(Z=0.017, Y=0.27)\end{array}$ & $0.83 \pm 0.01$ & $0.65 \pm 0.01$ \\
$\begin{array}{l}\text { Dotter et al. (2008) } \\
(Z=0.019, Y=0.27)\end{array}$ & $0.85 \pm 0.01$ & $0.66 \pm 0.01$ \\
$\begin{array}{l}\text { Bressan et al. }(2012) \\
(Z=0.017, Y=0.28)\end{array}$ & $0.83 \pm 0.01$ & $0.66 \pm 0.01$ \\
$\begin{array}{l}\text { Ekström et al. }(2012) \\
(Z=0.014, Y=0.27)\end{array}$ & $0.84 \pm 0.01$ & $0.64 \pm 0.01$ \\
$\begin{array}{l}\text { Baraffe et al. }(2015) \\
(Z=0.015, Y=0.27)\end{array}$ & $0.85 \pm 0.01$ & $0.67 \pm 0.01$ \\
\hline
\end{tabular}

Note. $Z=$ metallicity, $Y=$ He fraction.

\section{CONCLUSIONS}

Multi-epoch, infrared, AO imaging with LBTI/LMIRcam during the LEECH exoplanet imaging survey resolved the components of the known spectroscopic binary NO UMa at separations $<0$ " 09 . The binary exhibited about $180^{\circ}$ of orbital motion over the $\sim 7$ months spanning our images. We combined astrometry from our data with archival AO observations, published speckle interferometry measurements, and published velocity curves and performed a simultaneous astrometric/spectroscopic orbit fit using minimization techniques. The complete set of estimated orbital parameters from our fit are consistent with previous determinations that used data covering a smaller portion of the orbit and lead to significant improvements in precision. We estimated component masses of $0.83 \pm 0.02 M_{\odot}$ and $0.64 \pm 0.02 M_{\odot}$ for the primary and secondary, respectively. Our resolved, near-IR photometry, combined with a new system distance and empirical relations, revealed a $\mathrm{K} 2.0 \mathrm{~V} \pm 0.5$ primary and $\mathrm{K} 6.5 \mathrm{~V} \pm 0.5$ secondary. The distance and systemic velocity from our orbit fit was combined with binary motion corrected proper motions from the literature to revise the Galactic kinematics of NO UMa. We find that our kinematic estimates, that take binarity into account, are much improved over previous estimates that do not. This result suggests additional proposed binary UMaG members with large peculiar velocities can be reconciled if their kinematics are corrected for binary motion and strengthens NO UMa's status as a nuclear member of the $500 \pm 100 \mathrm{Myr}$ old UMaG. We compared our measured component masses to model estimates from five sets of modern stellar evolution models at the nominal age of the system and found excellent agreement between the measured and model masses with little scatter between the models. NO UMa joins the short list of bright, nearby, short period binaries with known ages and fully characterized orbits. It is thus a late-type, 


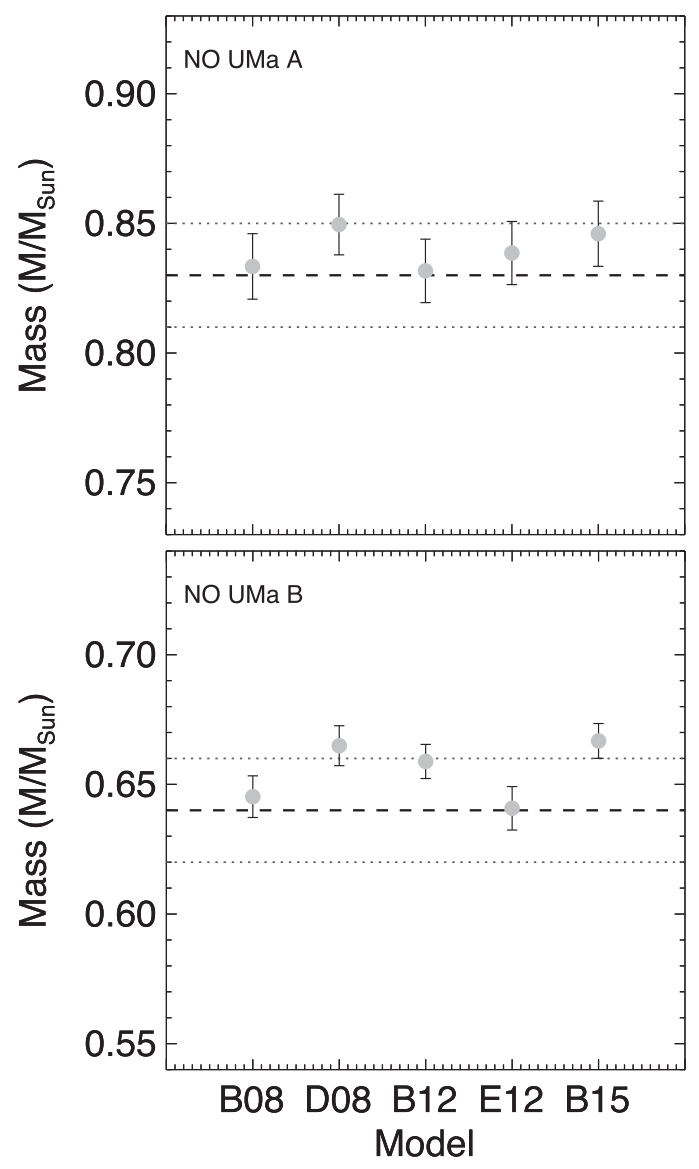

Figure 5. Measured masses (dashed) and uncertainties (dotted) of NO UMa A (top) and B (bottom) compared to estimated masses from five sets of stellar evolution models (filled circles) at an age of 500 Myr: (Bertelli et al. 2008, B08), (Dotter et al. 2008, D08), (Bressan et al. 2012, B12), (Ekström et al. 2012, E12), and (Baraffe et al. 2015, B15). The model estimated masses match our orbit derived masses well within the uncertainties and there is little scatter between the models.

mass and age benchmark. In addition to NO UMa, several other similar binaries have been resolved during LEECH observations and astrometric monitoring continues.

We thank the anonymous referee for their constructive review that improved the quality of this manuscript. We thank the LBTI/LMIRcam instrument team for providing support during LEECH observations. A portion of the research of J.E.S. was supported by an appointment to the NASA Postdoctoral Program at NASA Ames Research Center, administered by Oak Ridge Associated Universities through a contract with NASA. Support for A.J.S. was provided by the National Aeronautics and Space Administration through Hubble Fellowship grant HST-HF2-51349 awarded by the Space Telescope Science Institute, which is operated by the Association of Universities for Research in Astronomy, Inc., for NASA, under contract NAS 5-26555. A.-L.M. and S.D. acknowledge support from the "Progetti Premiali" funding scheme of the Italian Ministry of Education, University, and Research. E.B. is supported by the Swiss National Science Foundation (SNSF). LEECH is funded by the NASA Origins of Solar Systems Program, grant NNX13AJ17G. The Large Binocular Telescope Interferometer is funded by NASA as part of its Exoplanet Exploration program. LMIRcam is funded by the National Science Foundation through grant NSF AST-0705296.
STELLA was made possible by funding through the State of Brandenburg (MWFK) and the German Federal Ministry of Education and Research (BMBF). The facility is a collaboration of the AIP in Brandenburg with the IAC in Tenerife. This research has made use of the SIMBAD database, operated at CDS, Strasbourg, France. This publication makes use of data products from the Wide-field Infrared Survey Explorer, which is a joint project of the University of California, Los Angeles, and the Jet Propulsion Laboratory/California Institute of Technology, funded by the National Aeronautics and Space Administration. This publication makes use of data products from the Two Micron All Sky Survey, which is a joint project of the University of Massachusetts and the Infrared Processing and Analysis Center/California Institute of Technology, funded by the National Aeronautics and Space Administration and the National Science Foundation. This research has made use of the Keck Observatory Archive (KOA), which is operated by the W. M. Keck Observatory and the NASA Exoplanet Science Institute (NExScI), under contract with the National Aeronautics and Space Administration.

Facilities: LBT(LBTI/LMIRcam)

\section{APPENDIX ORBIT FIT FORMALISM}

In an astrometric (visual) binary system, the motion of the secondary relative to the primary is described by seven parameters called the Campbell elements: orbital period $P$, time of periastron passage $T_{0}$, semimajor axis $a$, eccentricity $e$, inclination $i$, argument of periastron $\omega$, and the position angle of the ascending node $\Omega$. If there exists a set of binary observations consisting of the time of observation $t$ and the relative coordinates of the secondary with respect to the primary $x, y$; and $P, T_{0}$, and $e$ are known, the remaining geometric elements of the orbit can be determined via minimization techniques through the Thiele-Innes elements: $A, B, F$, and $G$ (Hartkopf et al. 1989; Lucy 2013; Wöllert et al. 2014). This is possible because the Thiele-Innes elements are dependent on the orbital parameters:

$$
\begin{gathered}
A=a(\cos \omega \cos \Omega-\sin \omega \sin \Omega \cos i) \\
B=a(\cos \omega \sin \Omega+\sin \omega \cos \Omega \cos i) \\
F=a(-\sin \omega \cos \Omega-\cos \omega \sin \Omega \cos i) \\
G=a(-\sin \omega \sin \Omega+\cos \omega \cos \Omega \cos i) .
\end{gathered}
$$

In practice, $P, T_{0}$, and $e$ are not typically known a priori. Thus, grid search techniques (Hartkopf et al. 1989; Schaefer et al. 2006) or MC methods (Esposito et al. 2013) are used to explore the parameter space and determine initial guesses that are compatible with the observations. Then, for every set of $P, T_{0}$, and $e$, the eccentric anomaly $E$ is given by Kepler's equation,

$$
E-e \sin E=\frac{2 \pi}{P}\left(t-T_{0}\right),
$$

and $x$ and $y$ at time $t$ are dependent on the Thiele-Innes elements, $E$, and $e$,

$$
\begin{aligned}
& x=A \cdot X+F \cdot Y \\
& y=B \cdot X+G \cdot Y,
\end{aligned}
$$

where $X=\cos E-e$ and $Y=\sqrt{1-e^{2}} \cdot \sin E$. Determination of the geometric orbit parameters from the Thiele-Innes elements is then a problem of fitting a linear model to the 
observed data, minimizing the residuals between the model and observed $t, x$, and $y$, and solving the system of Thiele-Innes equations.

In a double-lined spectroscopic binary, velocity measurements of each component can independently yield $P, T_{0}, e$, and $\omega$. The inclusion of these data also adds three additional orbital parameters; the velocity semi-amplitudes of the primary and secondary, $K_{1}$ and $K_{2}$, respectively, and the systemicvelocity $\gamma$. The measured velocities of each component, $v_{1}$ and $v_{2}$, are given by

$$
\begin{aligned}
& v_{1}=+K_{1}[e \cos \omega+\cos (\nu+\omega)]+\gamma \\
& v_{2}=-K_{2}[e \cos \omega+\cos (\nu+\omega)]+\gamma,
\end{aligned}
$$

where the true anomaly $\nu$ can be calculated from the the orbital elements following,

$$
\tan \frac{\nu}{2}=\sqrt{\frac{1+e}{1-e}} \tan \frac{E}{2} .
$$

Thus, the addition of spectroscopic velocity data and a simultaneous astrometric/spectroscopic orbit fit allows determination of all 10 orbital elements $\left(P, T_{0}, e, a, i, \omega, \Omega, K_{1}, K_{2}\right.$, $\gamma)$ and a full orbit solution (Schaefer et al. 2008). The resulting astrometric and spectroscopic orbital parameters provide the masses of the primary and secondary

$$
\begin{aligned}
& M_{1}=\frac{1.036 \times 10^{-7}\left(K_{1}+K_{2}\right)^{2} K_{2} P\left(1-e^{2}\right)^{3 / 2}}{\sin ^{3} i} \\
& M_{2}=\frac{1.036 \times 10^{-7}\left(K_{1}+K_{2}\right)^{2} K_{1} P\left(1-e^{2}\right)^{3 / 2}}{\sin ^{3} i},
\end{aligned}
$$

and the distance to the system

$$
d=\frac{9.189 \times 10^{-5}\left(K_{1}+K_{2}\right) P\left(1-e^{2}\right)^{1 / 2}}{a \sin i},
$$

where $K_{1}$ and $K_{2}$ are in $\mathrm{km} \mathrm{s}^{-1}, P$ is in days, and $a$ is in arcseconds and $M_{1}$ and $M_{2}$ are in $M_{\odot}$ and $d$ is in pc (Schaefer et al. 2008).

\section{REFERENCES}

Allende Prieto, C. 2004, AN, 325, 604

Ammler-von Eiff, M., \& Guenther, E. W. 2009, A\&A, 508, 677

Arenou, F., Halbwachs, J.-L., Mayor, M., Palasi, J., \& Udry, S. 2000, in IAU

Symp. 200, The Formation of Binary Stars, ed. B. Reipurth \& H. Zinnecker (San Francisco, CA: ASP), 135

Bailey, V. P., Hinz, P. M., Puglisi, A. T., et al. 2014, Proc. SPIE, 9148, 3

Balega, I. I., Balega, Y. Y., Gasanova, L. T., et al. 2013, AstBu, 68, 53

Baraffe, I., Chabrier, G., Allard, F., \& Hauschildt, P. H. 1998, A\&A, 337, 403

Baraffe, I., Homeier, D., Allard, F., \& Chabrier, G. 2015, A\&A, 577, A42

Baranne, A., Mayor, M., \& Poncet, J. L. 1979, VA, 23, 279

Beckers, J. M. 1993, ARA\&A, 31, 13

Bertelli, G., Girardi, L., Marigo, P., \& Nasi, E. 2008, A\&A, 484, 815

Beuzit, J.-L., Feldt, M., Dohlen, K., et al. 2008, Proc. SPIE, 7014, 18

Bonnefoy, M., Currie, T., Marleau, G.-D., et al. 2014, A\&A, 562, A111

Bowler, B. P., Liu, M. C., Shkolnik, E. L., \& Tamura, M. 2015, ApJS, 216, 7

Brandt, T. D., \& Huang, C. X. 2015, arXiv:1501.04404

Bressan, A., Marigo, P., Girardi, L., et al. 2012, MNRAS, 427, 127

Burrows, A., Marley, M., Hubbard, W. B., et al. 1997, ApJ, 491, 856

Cutri, R. M., Skrutskie, M. F., van Dyk, S., et al. 2003, 2MASS All Sky Catalog of Point Sources

Cutri, R. M., et al. 2013, yCat, 2328, 0

Delorme, P., Gagné, J., Girard, J. H., et al. 2013, A\&A, 553, L5

Dotter, A., Chaboyer, B., Jevremović, D., et al. 2008, ApJS, 178, 89

Duchêne, G., \& Kraus, A. 2013, ARA\&A, 51, 269
Duquennoy, A., Mayor, M., \& Halbwachs, J.-L. 1991, A\&AS, 88, 281

Dutrey, A., di Folco, E., Guilloteau, S., et al. 2014, Natur, 514, 600

Ekström, S., Georgy, C., Eggenberger, P., et al. 2012, A\&A, 537, A146

Esposito, S., Riccardi, A., Pinna, E., et al. 2011, Proc. SPIE, 8149, 814902

Esposito, S., Riccardi, A., Quirós-Pacheco, F., et al. 2010, ApOpt, 49, G174

Esposito, S., Mesa, D., Skemer, A., et al. 2013, A\&A, 549, A52

Goldin, A., \& Makarov, V. V. 2007, ApJS, 173, 137

Gontcharov, G. A. 2006, AstL, 32, 759

Halbwachs, J., Arenou, F., Mayor, M., \& Udry, S. 2000, in IAU Symp. 200, The Formation of Binary Stars, ed. B. Reipurth \& H. Zinnecker (San Francisco, CA: ASP), 132

Halbwachs, J. L., Mayor, M., Udry, S., \& Arenou, F. 2003, A\&A, 397, 159

Hartkopf, W. I., McAlister, H. A., \& Franz, O. G. 1989, AJ, 98, 1014

Heinze, A. N., \& Hinz, P. M. 2005, AJ, 130, 1929

Hinkley, S., Oppenheimer, B. R., Zimmerman, N., et al. 2011, PASP, 123, 74

Hinz, P. M., Bippert-Plymate, T., Breuninger, A., et al. 2008, Proc. SPIE, 7013, 28

Høg, E., Fabricius, C., Makarov, V. V., et al. 2000, A\&A, 355, L27

Johnson, D. R. H., \& Soderblom, D. R. 1987, AJ, 93, 864

Johnson, H. L., \& Morgan, W. W. 1953, ApJ, 117, 313

Jovanovic, M., Weber, M., \& Allende Prieto, C. 2013, POBeo, 92, 169

King, J. R., \& Schuler, S. C. 2005, PASP, 117, 911

King, J. R., Villarreal, A. R., Soderblom, D. R., Gulliver, A. F., \& Adelman, S. J. 2003, AJ, 125, 1980

Kley, W., \& Haghighipour, N. 2014, A\&A, 564, A72

Kraus, A. L., Ireland, M. J., Cieza, L. A., et al. 2014, ApJ, 781, 20

Leisenring, J. M., Skrutskie, M. F., Hinz, P. M., et al. 2012, Proc. SPIE, 8446, $84464 \mathrm{~F}$

Lucy, L. B. 2013, A\&A, 551, A47

Macintosh, B. A., Graham, J. R., Palmer, D. W., et al. 2008, Proc. SPIE, 7015,18

Maire, A.-L., Skemer, A. J., Hinz, P. M., et al. 2015, A\&A, 576, A133

Mamajek, E. E., \& Hillenbrand, L. A. 2008, ApJ, 687, 1264

Mamajek, E. E., Kenworthy, M. A., Hinz, P. M., \& Meyer, M. R. 2010, AJ, 139,919

Marleau, G.-D., \& Cumming, A. 2014, MNRAS, 437, 1378

Mayor, M., Duquennoy, A., Halbwachs, J.-L., \& Mermilliod, J.-C. 1992, in ASP Conf. Ser. 32, IAU Coll. 135: Complementary Approaches to Double and Multiple Star Research, ed. H. A. McAlister \& W. I. Hartkopf (San Francisco, CA: ASP), 73

Mermilliod, J.-C., \& Mermilliod, M. 1994, Catalogue of Mean UBV Data on Stars

Pecaut, M. J., \& Mamajek, E. E. 2013, ApJS, 208, 9

Perryman, M. A. C., Lindegren, L., Kovalevsky, J., et al. 1997, A\&A, 323, L49 Proctor, R. A. 1869, RSPS, 18, 169

Raghavan, D., McAlister, H. A., Henry, T. J., et al. 2010, ApJS, 190, 1

Rapson, V. A., Kastner, J. H., Andrews, S. M., et al. 2015, ApJL, 803, L10

Riccardi, A., Xompero, M., Briguglio, R., et al. 2010, Proc. SPIE, 7736, 2

Riedel, A. R., Finch, C. T., Henry, T. J., et al. 2014, AJ, 147, 85

Roman, N. G. 1949, ApJ, 110, 205

Schaefer, G. H., Simon, M., Beck, T. L., Nelan, E., \& Prato, L. 2006, AJ, 132,2618

Schaefer, G. H., Simon, M., Prato, L., \& Barman, T. 2008, AJ, 135, 1659

Schlieder, J. E., Bonnefoy, M., Herbst, T. M., et al. 2014, ApJ, 783, 27

Shkolnik, E. L., Anglada-Escudé, G., Liu, M. C., et al. 2012, ApJ, 758, 56

Skemer, A. J., Hinz, P., Esposito, S., et al. 2014a, Proc. SPIE, 9148, 0

Skemer, A. J., Hinz, P. M., Esposito, S., et al. 2012, ApJ, 753, 14

Skemer, A. J., Marley, M. S., Hinz, P. M., et al. 2014b, ApJ, 792, 17

Skrutskie, M. F., Jones, T., Hinz, P., et al. 2010, Proc. SPIE, 7735, 77353H

Soderblom, D. R., \& Mayor, M. 1993, AJ, 105, 226

Spiegel, D. S., \& Burrows, A. 2012, ApJ, 745, 174

Strassmeier, K., Washuettl, A., Granzer, T., Scheck, M., \& Weber, M. 2000, A\&AS, 142, 275

Strassmeier, K. G., Weber, M., Granzer, T., \& Järvinen, S. 2012, AN, 333, 663

Tabernero, H. M., Montes, D., Gonzalez Hernandez, J. I., \& Ammler-von Eiff, M. 2014, arXiv:1409.2348

Tang, Y.-W., Dutrey, A., Guilloteau, S., et al. 2014, ApJ, 793, 10

Thalmann, C., Desidera, S., Bonavita, M., et al. 2014, A\&A, 572, A91

van Leeuwen, F. 2007, A\&A, 474, 653

Weber, M., \& Strassmeier, K. G. 2011, A\&A, 531, A89

Welsh, W. F., Orosz, J. A., Short, D. R., et al. 2015, ApJ, 809, 26

Wizinowich, P., Acton, D. S., Shelton, C., et al. 2000, PASP, 112, 315

Wöllert, M., Brandner, W., Reffert, S., et al. 2014, A\&A, 564, A10

Wright, E. L., Eisenhardt, P. R. M., Mainzer, A. K., et al. 2010, AJ, 140, 1868

Yoss, K. M. 1961, ApJ, 134, 809 